\title{
LA FACILITACIÓN COMO UN MECANISMO QUE INCREMENTA LA DIVERSIDAD VEGETAL EN AMBIENTES EXTREMOS
}

\author{
FACILITATION AS A MECHANISM TO INCREASE PLANT DIVERSITY IN EXTREM \\ ENVIRONMENTS
}

\author{
Nilsen L. Lasso-Rivas
}

\begin{abstract}
RESUMEN
Tradicionalmente se consideró que las interacciones negativas entre especies de plantas eran la fuerza modeladora de la estructura y el funcionamiento de los ecosistemas, mientras las interacciones positivas eran de poca importancia en ese sentido. Dicha visión ha cambiado en las últimas décadas y actualmente se considera que las interacciones de tipo positivo, también juegan un papel importante para la estructuración y el funcionamiento de los ecosistemas. La facilitación es un tipo de interacción positiva no trófica, que se produce entre plantas fisiológicamente independientes y que está mediada a través de cambios en el entorno abiótico o a través de otros organismos. La mayor parte de la investigación realizada en el campo de la facilitación en los últimos veinte años se ha desarrollado a partir de la hipótesis del gradiente de estrés (HGE). La facilitación puede actuar directa o indirectamente a través de diferentes mecanismos. Los resultados de múltiples investigaciones, indican que la facilitación puede aumentar la diversidad de las especies vegetales en ambientes extremos, donde la presencia de una especie nodriza modifica el ambiente de manera tal, que resulta en una reducción en la frecuencia e intensidad de alguna perturbación física o estrés, permitiendo que las especies menos tolerantes puedan sobrevivir. Sin embargo, existen pocos estudios que cuantifiquen el número de especies que se agregan a una comunidad debido a la facilitación, así el reto es diseñar experimentos y estudios que permitan cuantificar la importancia relativa de la facilitación para el mantenimiento de la biodiversidad en ecosistemas extremos.
\end{abstract}

PALABRAS CLAVE: interacción planta-planta, estrés abiótico, comunidad vegetal

\begin{abstract}
Negative interactions among plants are considered the most important factors determining the shape, structure and dynamics of plant communities, and because of this viewpoint, much research has been devoted to understanding the nature of competitive interactions among plants. However, the result of multiple studies in the last two decades had shown that positive interaction could also play an important role determining the structure and function of terrestrial ecosystems. Facilitation is a type of nontrophic positive interaction that occurs between physiologically independent individuals and is mediated through changes in the abiotic environment or through other organisms. Most of the research in the field of facilitation in the last twenty years has been developed from the stress gradient hypothesis (SGH). Facilitation may act directly or indirectly through different mechanisms. The results of multiple investigations indicate that facilitation can increase the diversity of plant species in harsh environments, where facilitator species can mitigate extreme conditions and benefit other species. Still, few studies have quantified the number of species that are added to a community because facilitation and the challenge is to design experiments and studies to quantify the relative importance of the provision for the maintenance of biodiversity in extreme environments.
\end{abstract}

KEY WORDS: plant-plant interactions, abiotic stress, plant community 


\section{INTRODUCCIÓN}

Tratar de comprender la naturaleza de las interacciones entre las plantas, ha sido fundamental en ecología vegetal y cobra mayor importancia en la actualidad, pues los resultados de las investigaciones sobre las interacciones entre plantas, pueden ayudar a mejorar la comprensión de las respuestas de las comunidades vegetales al cambio climático (Michalet et al., 2006; Stiling y Cornelissen, 2007). Existen muchos tipos de interacción entre las plantas, algunas de ellas negativas (ej. parasitismo, competencia, y alelopatía) y otras positivas (ej. facilitación y mutualismo). Aunque las interacciones negativas, tradicionalmente han sido consideradas como la fuerza que moldea la estructura y el funcionamiento de los ecosistemas, los resultados de múltiples estudios en las dos últimas décadas sobre el papel de las interacciones positivas en las comunidades vegetales, han demostrado que juegan un papel tan importante como el de las interacciones negativas (Callaway et al., 2002; Brooker et al., 2008). El mutualismo se define como interacciones positivas de tipo recíproco entre pares de especies (Bronstein, 2009) y la facilitación como una interacción no trófica, que se produce entre plantas fisiológicamente independientes y que está mediada a través de cambios en el entorno abiótico o a través de otros organismos (Brooker et al., 2008). Puesto que el papel que desempeñan las interacciones positivas no tróficas en las comunidades vegetales no es ampliamente reconocido, el objetivo de la presente contribución es el de explorar los mecanismos de la facilitación y sus efectos en la diversidad vegetal.

\section{INTERACCIONES POSITIVAS}

El mutualismo y la facilitación comparten la característica de que su acción se da mediante la disminución de los efectos negativos que produce estrés en las plantas, definido el término "estrés" como aquellos factores que limitan la tasa de producción de materia seca (Grace, 1991). Es interesante que la mayoría de los estudios sobre la facilitación entre plantas se han hecho en ambientes extremos tales como desiertos (Tewksbury y Lloyd, 2001; Tirado y Pugnaire, 2003; Flores y Jurado, 2003), montañas mediterráneas (Gómez-Aparicio et al., 2004) y estepas alpinas (Callaway et al., 2002). Igualmente, la mayor parte de estas investigaciones en los últimos veinte años ha sido estimulada por la hipótesis del gradiente de estrés (HGE). Esta hipótesis propone que un incremento en el estrés biótico provoca un aumento en la facilitación respecto a las competencias. Entonces es de esperar que la facilitación sea más importante cuando las plantas crecen en ambientes severos (Callaway, 2007).

Si bien la HGE ha generado el aumento significativo en el conocimiento de las interacciones positivas entre plantas, también se he debatido activamente durante la última década acerca de cómo las interacciones entre plantas cambian a lo largo de gradientes ambientales. Por ejemplo, los resultados de un meta análisis de estudios de interacciones entre plantas en ambientes áridos, indican que la facilitación no parece aumentar a medida que las condiciones ambientales se vuelven más estresantes (Maestre y Cortina, 2004; Maestre et al., 2005). Otros investigadores sugieren que la facilitación puede ser más importante a niveles de estrés intermedio (Holmgren y Scheffer, 2010). Algunos autores plantean que estos puntos de vista, aparentemente opuestos, se pueden conciliar mediante la estandarización de términos tales como nicho fundamental y nicho realizado, además de la evaluación crítica de la funcionalidad de los gradientes de estrés, la evaluación de la capacidad competitiva y la tolerancia al estrés de las especies en una comunidad, también de la clarificación de la escala en la que se enfoca cada estudio, la definición del concepto de estrés empleado por el autor y si este concepto aplica únicamente a las especies en particular o para la comunidad cuyas especies pueden diferir ampliamente en sus adaptaciones ecológicas (Maestre et al., 2009; He y Bertness, 2014; Soliveres et al., 2015).

\section{MECANISMOS DE LA FACILITACIÓN}

La facilitación puede actuar directamente o indirectamente a través de diferentes mecanismos. De manera directa ocurre cuando una planta modifica las condiciones físicas de un sitio, lo que resulta en un beneficio para una planta vecina, así pues, la facilitación directa implica la protección contra condiciones ambientales adversas (Brooker, 2006). El fenómeno de la facilitación directa también es conocido como el síndrome de la planta nodriza (Ren et al., 2008); estas plantas facilitan el crecimiento y el desarrollo de otras especies de plantas bajo su dosel, estás son conocidas como especies objetivo, ya que ofrecen microhábitats, que son favorables para la germinación de semillas y/o el 
reclutamiento de plántulas ((Bruno et al., 2003; Padilla y Pugnaire, 2006; Ren et al., 2008). Por ejemplo, Franco y Nobel (1989) encontraron en el desierto de Sonora en México, que el establecimiento de plántulas de la especie de cactus Carnegiea gigantia, se facilitaba cuando crecían bajo el dosel de las especies arbustivas, Ambrosia deltoidea y Cercidium microphyllum; por la reducción de la temperatura de superficie del suelo y al proveer un microhábitat, con altos niveles de nitrógeno. Igualmente, Suzán et al. (1996) registraron en el desierto de Sonora en Arizona EE.UU., la acción facilitadora del arbusto Olneya tesota, la cual crea bajo su dosel un microhábitat adecuado para el establecimiento de más de 75 especies de plantas nativas de esa zona. Otros estudios sugieren un papel facilitador de las plantas en cojín en los hábitats alpinos. Dichas plantas facilitan el establecimiento para otras especies que crecen en su interior debido a la morfología compacta del cojín que atenúa el efecto de las condiciones ambientales extremas (Nuñez et al., 1999; Cavieres et al., 2006; Anthelme et al., 2014). Otro tipo de facilitación directa lo constituyen las denominadas especies modificadoras de hábitat, estas especies cambian las condiciones abióticas de un sitio, de forma que afectan la distribución y abundancia de otras especies (Badano et al., 2007). Por ejemplo, Ammophila arenaria es una especie pionera en la colonización de las dunas templadas en el hemisferio norte, que tolera altas concentraciones de sal, puede sobrevivir al ser tapada por la arena y hace simbiosis con hongos micorrízico arbusculares (Kowalchuk et al., 1997) estas características le permiten establecerse en condiciones extremadamente pobres en nutrientes y modificar el hábitat lo que facilita el establecimiento de especies de etapas de sucesión tardía que no pueden colonizar el ambiente original de la playa (Huiskes, 1979).

La interacción de facilitación entre dos especies se considera indirecta, cuando es mediada por una tercera especie, este tipo de interacción solo se produce cuando más de dos especies están presentes (Brooker et al., 2008). La facilitación indirecta puede darse a través de mecanismos como el denominado efecto imán, la resistencia asociativa, y el intercambio de recursos a través de redes comunes de micorrizas. En el efecto imán, las plantas que carecen de recompensas florales, pueden atraer a los polinizadores al compartir señales florales con plantas que si producen este tipo de recompensas. Por ejemplo, Laverty (1992) estudió la producción de frutos y semillas en Podophyllum peltatum, una hierba nativa del este de Norteamérica que no producen néctar, pero dependen de las visitas infrecuentes de abejorros que buscan el néctar de otras especies. Los resultados de la investigación mostraron que el éxito reproductivo de $P$. peltatun, se incrementó con la proximidad de Pedicularis canadensis, un productor prolífico de néctar muy visitado por los abejorros. De manera similar, Johnson et al. (2003) evaluaron el éxito de polinización de la orquídea Anacamptis morio, esta orquídea es polinizada por abejorros, pero no ofrece recompensa alguna. Los investigadores encontraron que la planta tenía un mayor éxito de polinización, cuando los individuos eran trasplantados cerca de plantas productoras de néctar que cuando eran trasplantadas lejos de éstas. En la resistencia asociativa, el establecimiento de una especie se ve favorecido por la intervención de una planta nodriza que la protege de la herbivoría, esto puede darse a través de defensas físicas o químicas (Rousset y Lepart, 2000; Smit et al., 2005). Por ejemplo, Smit et al. (2006) evaluaron el efecto del pastoreo en el establecimiento de plántulas del genero Picea y encontraron que los individuos que crecían cerca de plantas con sabor desagradable, sufrían menor herbívora y tenían una tasa de sobrevivencia mayor que aquellos que crecían cerca de plantas con sabor agradable. Finalmente, el intercambio de recursos a través de redes comunes de micorrizas permite la transferencia de recursos entre plantas ya establecidas y plántulas. Este flujo de recursos aumenta de manera significativa la probabilidad de éxito en el establecimiento de las nuevas plántulas (Teste et al., 2009).

\section{LA FACILITACIÓN Y LA DIVERSIDAD DE ESPECIES}

La facilitación puede aumentar la diversidad, cuando la presencia de una especie modifica el medio ambiente de manera tal, que reduce la frecuencia e intensidad de alguna perturbación física o algún factor de estrés, lo que permite que especies menos tolerantes puedan sobrevivir (Hacker y Gaines, 1997; Cavieres y Badano, 2010; McIntire y Fajardo, 2014). Los estudios que muestran el efecto de la facilitación en la diversidad de especies vegetales, se basan en comparaciones entre la riqueza de especies que crecen bajo el dosel de plantas nodrizas y las especies que crecen por fuera del dosel. La mayoría de dichos estudios muestran mayor riqueza de especies bajo el dosel de las especies nodrizas, 
en comparación con las áreas abiertas. Por ejemplo, Raffaele y Veblen (1998) en el norte de la Patagonia Argentina, evaluaron la influencia de dos especies de arbustos (Schinus patagonicus y Berberis buxifolia) en la supervivencia, crecimiento, y reproducción vegetativa de plantas herbáceas y leñosas, en un matorral sujeto a eventos de fuego. Encontraron que la abundancia de rebrotes así como el número de especies, era mayor debajo de los arbustos intactos, que bajo los arbustos sometido a poda. De manera similar, Larrea-Alcázar et al. (2005), evaluaron el papel de Prosopis flexuosa en el establecimiento de otras especies vegetales en un valle semiárido de Bolivia y encontraron un mayor número de especies de asteráceas debajo el dosel de $P$. flexuosa que en espacios abiertos. Igualmente, Badano et al. (2006) analizaron el efecto de la planta en cojín Oreopolus glacialis sobre la riqueza, la abundancia, la diversidad y la equitatividad, en una comunidad vegetal de la alta montaña en la cordillera de los Andes, y determinaron que la riqueza de especies y la abundancia de individuos por unidad de área, era mayor al interior de los cojines que en los espacios abiertos. Finalmente, van Zonneveld et al. (2012), encontraron que los arbustos nodriza aumentaban la diversidad y abundancia de plántulas a lo largo del límite entre un matorral árido y un bosque pluvial templado en el norte de Chile. Sin embargo, algunos estudios muestran una riqueza de especies similar o menor debajo el dosel de las plantas nodrizas, como es el caso de Porlieria. chilensis (Gutiérrez et al., 1993). De forma similar, De Villiers et al. (2001), encontraron que la riqueza de especies y la densidades de plántulas era significativamente mayor en las áreas abiertas que debajo de los arbustos; igualmente, Rossi y Villagra, (2003), al analizar el efecto de Prosopis flexuosa en la composición y abundancia de arbustos y herbáceas para un sitio desértico en Chile, encontraron un menor número de especie bajo el dosel de $P$. flexuosa que en las zonas abiertas. Estos resultados contrastantes parecerían indicar que el efecto facilitador de algunas especies no genera necesariamente un aumento en la riqueza de especies. Sin embargo, puesto que las plantas nodrizas y el hábitat adyacente hacen parte de la misma comunidad y que las plantas nodrizas favorecen el establecimiento de especies, que de otro modo serian excluidas de la comunidad, genera un aumento neto en la diversidad. Vale la pena anotar que algunos autores indican que las especies nodrizas podrían incrementar la diversidad filogenética en las comunidades vegetales (Valiente-Banuet y Verdú, 2007). Pocos estudios han intentado cuantificar el número de especies que se agregan a una comunidad debido a la facilitación. Uno de los pocos esfuerzos en este sentido mostró que, en un pantano salobre, al menos el $35 \%$ de la diversidad de especies observada estaba presente como resultado de la facilitación (Hacker y Gaines, 1997). Así pues, el reto es tratar de cuantificar la importancia relativa de la facilitación para el mantenimiento de la biodiversidad en diferentes ecosistemas mediante estudios que puedan detectar interacciones positivas, para luego evaluar su importancia relativa a escala local y global.

\section{CONCLUSIONES}

Aunque tradicionalmente las interacciones positivas no han sido consideradas como un factor importante para la estructura y función de las comunidades vegetales, los resultados de las investigaciones de las dos últimas décadas sugieren lo contrario. La facilitación puede actuar de manera directa mediante mecanismos como el síndrome de la planta nodriza y las especies modificadoras de hábitat, también puede actuar de manera indirecta cuando es mediada por una tercera especie, a través de mecanismos como el efecto imán, la resistencia asociativa y el intercambio de recursos a través de redes comunes de micorrizas. La evidencia reciente indica que la facilitación puede tener un efecto positivo en la diversidad de las comunidades en ambientes severos, sin embargo, los estudios que abordan este tema son escasos y hace falta cuantificar la importancia relativa de la facilitación para el mantenimiento de la biodiversidad mundial, así pues se deben diseñar experimentos y estudios que contribuyan con el entendimiento de este proceso.

\section{AGRADECIMIENTOS}

Expreso mis agradecimientos al Programa de Agronomía de la Universidad del Pacífico por su continuo apoyo.

\section{BIBLIOGRAFÍA}

Anthelme, F., Cavieres, L.A. y Dangles, O. 2014. Facilitation among plants in alpine environments in the face of climate change. Frontiers in Plant Science, 5: 387.

Badano, E.I., Villarroel, E., Bustamante, R.O., Marquet, P.A. y Cavieres, L.A. 2007. Ecosystem engineering facilitates invasions by exotic plants in high-Andean ecosystems. Journal of Ecology 95: 682-688. 
Badano, E., Jones, C.G., Cavieres, L.A. y Wright. J.P. 2006. Assessing impacts of ecosystem engineers on community organization: a general approach illustrated by effects of a high-Andean cushion plant. Oikos 115:369-385.

Bertness, M. y Ewanchuk. P. 2002. Latitudinal and climatedriven variation in the strength and nature of biological interactions in New England salt marshes. Oecologia 132:392-401.

Bronstein, J.L. 2009. The evolution of facilitation and mutualism. Journal of Ecology 97:1160-1170.

Brooker, R.W. 2006. Plant-plant interactions and environmental change. The New phytologist 171:271-84.

Brooker, R.W., Maestre, F.T., Callaway, R.M., Lortie, C.L., Cavieres, L.A., Kunstler, G., Liancourt, P., Tielbörger, K., Travis, J.M.J., Anthelme, F., Armas, C., Coll, L., Corcket, E., Delzon, S., Forey, E., Kikvidze, Z., Olofsson, J., Pugnaire, F., Quiroz, C. L., Saccone, P., Schiffers, K., Seifan, M., Touzard, B. y Michalet, R. 2008. Facilitation in plant communities: the past, the present, and the future. Journal of Ecology 96:18-34.

Bruno, J.F., Stachowicz, J.J. y Bertness, M.D. 2003. Inclusion of facilitation into ecological theory. Trends in Ecology y Evolution 18:119-125.

Callaway, R.M. 2007. Positive Interactions and Interdependence in Plant Communities. Springer.

Callaway, R.M., Brooker, R.W., Choler, P., Kikvidze, Z., Lortie, C.J., Michalet, R., Paolini, L., Pugnaire, F.I., Newingham, B., Aschehoug, E.T., Armas, C., Kikodze, D. y Cook, B.J. 2002. Positive interactions among alpine plants increase with stress. Nature 417:844-8.

Castanho, C. de T., y Prado, P. I. 2014. Benefit of shading by nurse plant does not change along a stress gradient in a coastal dune. PloS one 9:e105082.

Cavieres, L.A. y Badano, E.I. 2010. Consequences of facilitation on species diversity in terrestrial plant communities. In: Pugnaire, F.I. (ed.) Positive plant interactions and community dynamics. pp. 39-57. CRC Press, Boca Raton, FL, US.

Cavieres, L.A., Badano, E.I., Sierra-Almeida, A., GómezGonzález, S. y Molina-Montenegro, M.A. 2006. Positive interactions between alpine plant species and the nurse cushion plant Laretia acaulis do not increase with elevation in the Andes of central Chile. The New phytologist 169:59-69.
Flores, J., y Jurado, E. 2003. Are nurse-protégé interactions more common among plants from arid environments? Journal of Vegetation Science 14:911-916.

Franco, A., y Nobel, P. 1989. Effect of nurse plants on the micro-habitat and growth of cacti. Journal of Ecology 77:870-886.

Franks, S.J. 2003. Facilitation in multiple life-history stages: evidence for nucleated succession in coastal dunes. Plant Ecology 168:1-11.

Gómez-Aparicio, L., Zamora, R., Gómez, J.M., Hódar, J.A., Castro, J. y Baraza, E. 2004. Applying plant facilitation to forest restoration: a meta-analysis of the use of shrubs as nurse plants. Ecological Applications 14:1128-1138.

Grace, J.B. 1991. A Clarification of the Debate Between Grime and Tilman. Functional Ecology 5:583.

Gutiérrez, J. R., Meserve, P.L., Contreas, L.C., Vásquez, H. y Jaksic, F.M. 1993. Spatial distribution of soil nutrients and ephemeral plants underneath and outside the canopy of Porlieria chilensis shrubs (Zygophyllaceae) in arid coastal Chile. Oecologia 95:347-352.

Hacker, S.D. y Gaines, S.D. 1997. Some implications of direct positive interactions for community species diversity. Ecology 78:1990-2003.

He, Q. y Bertness, M.D. 2014. Extreme stresses, niches, and positive species interactions along stress gradients. Ecology 95:1437-1443.

Holmgren, M. y Scheffer, M. 2010. Strong facilitation in mild environments: the stress gradient hypothesis revisited. Journal of Ecology 98:1269-1275.

Huiskes, A.H.L. 1979. Damage to marram grass Ammophila arenaria by larvae of Meromyza pratorum (Diptera). Ecography 2:182-185.

Johnson, S.D., Peter, C.I., Nilsson, L.A. y Ågren, J. 2003. Pollination success in a deceptive orchid is enhanced by co-occurring rewarding magnet plants. Ecology 84:2919-2927.

Kowalchuk, G.A., Gerards, S. y W. Woldendorp, J. 1997. Detection and characterization of fungal infections of Ammophila arenaria (marram grass) roots by denaturing gradient gel electrophoresis of specifically amplified 18s rDNA. Applied and environmental microbiology 63:3858-65. 
Larrea-Alcázar, D., López, R.P. y Barrientos, D. 2005. The nurse-plant effect of Prosopis flexuosa D.C. (LeguminosaeMimosoideae) in a dry valley of the Bolivian Andes 18:89-95.

Laverty, T.M. 1992. Plant interactions for pollinator visits: a test of the magnet species effect. Oecologia 89:502-508.

Maestre, F.T., Callaway, R.M., Valladares, F. y Lortie, C.J. 2009. Refining the stress-gradient hypothesis for competition and facilitation in plant communities. Journal of Ecology 97: 199-205.

Maestre, F.T., Valladares, F. y Reynolds, J.F. 2005. Is the change of plant-plant interactions with abiotic stress predictable? A meta-analysis of field results in arid environments. Journal of Ecology 93:748-757.

Maestre, F.T. y Cortina, J. 2004. Do positive interactions increase with abiotic stress? A test from a semi-arid steppe. Proceedings of the Royal Society B: Biological Sciences 271: S331- S333.

McIntire, E.J.B. y Fajardo, A. 2014. Facilitation as a ubiquitous driver of biodiversity. New Phytol, 201: 403-416.

Michalet, R., Brooker, R.W., Cavieres, L.A., Kikvidze, Z., Lortie, C.J., Pugnaire, F.I., Valiente-Banuet, A. y Callaway, R.M. 2006. Do biotic interactions shape both sides of the humped-back model of species richness in plant communities? Ecology letters 9:767-73.

Nuñez, C.I., Aizen, M.A., y Ezcurra, C. 1999. Species associations and nurse plant effects in patches of high-Andean vegetation. Journal of Vegetation Science 10:357-364.

Padilla, F.M. y Pugnaire, F.I. 2006. The role of nurse plants in the restoration of degraded environments. Frontiers in Ecology and the Environment 4:196-202.

Raffaele, E. y Veblen, T.T. 1998. Facilitation by nurse shrubs of resprouting behavior in a post-fire shrubland in northern Patagonia, Argentina. Journal of Vegetation Science 9:693-698.

Ren, H., Yang, L. y Liu, N. 2008. Nurse plant theory and its application in ecological restoration in lower subtropics of 98 China. Progress in Natural Science 18:137-142.

Rossi, B.E. y Villagra, P.E. 2003. Effects of Prosopis flexuosa on soil properties and the spatial pattern of understorey species in arid Argentina. Journal of Vegetation Science 14:543-550.

Rousset, O. y Lepart, J. 2000. Positive and negative interactions at different life stages of a colonizing species (Quercus humilis). Journal of Ecology 88:401-412.

Shumway, S.W. 2000. Facilitative effects of a sand dune shrub on species growing beneath the shrub canopy. Oecologia 124:138-148.

Smit, C., Béguin, D., Buttler, A. y Müller-Schärer, H. 2005. Safe sites for tree regeneration in wooded pastures: A case of associational resistance? Journal of Vegetation Science 16:209-214.

Smit, C., Den Ouden, J. y Muller-Scharer, H. 2006. Unpalatable plants facilitate tree sapling survival in wooded pastures. Journal of Applied Ecology 43:305-312.

Soliveres, S., Smit, C. y Maestre, F.T. 2015. Moving forward on facilitation research: response to changing environments and effects on the diversity, functioning and evolution of plant communities. Biological reviews of the Cambridge Philosophical Society 90:297-313.

Stiling, P. y Cornelissen, T. 2007. How does elevated carbon dioxide (CO 2) affect plant-herbivore interactions? A field experiment and meta-analysis of $\mathrm{CO} 2$-mediated changes on plant chemistry and herbivore performance. Global Change Biology 13:1823-1842.

Suzán, H., Nabhan, G.P. y Patten, D.T. 1996. The importance of Olneya tesota as a nurse plant in the Sonoran Desert. Journal of Vegetation Science 7:635-644.

Teste, F.P., Simard, S.W., Durall, D.M., Guy, R.D., Jones, M.D. y Schoonmaker, A.L. 2009. Access to mycorrhizal networks and roots of trees: importance for seedling survival and resource transfer. Ecology 90:2808-2822.

Tewksbury J. y Lloyd D. 2001. Positive interactions under nurse-plants: spatial scale, stress gradients and benefactor size. Oecologia 127:425-434.

Tirado, R. y Pugnaire, F.I. 2003. Shrub spatial aggregation and consequences for reproductive success. Oecologia 136:296-301.

Valiente-Banuet, A., y Verdú, M. 2007. Facilitation can increase the phylogenetic diversity of plant communities. Ecology letters 10:1029-36. 
De Villiers, A.J., Van Rooyen, M.W. y Theron, G.K. 2001. The role of facilitation in seedling recruitment and survival patterns, in the Strandveld Succulent Karoo, South Africa. Journal of Arid Environments 49:809-821. van Zonneveld, M.J., Gutiérrez, J.R. y Holmgren, M. 2012. Shrub facilitation increases plant diversity along an arid scrubland-temperate rain forest boundary in South America. Journal of Vegetation Science 23:541-551.

Fecha de recepción: 15/09/2015

Fecha de aceptación: 05/11/2015

Para citar este artículo: Lasso-Rivas, N. 2015. La facilitación como un mecanismo que incrementa la diversidad vegetal en ambientes extremos. Revista Intropica Vol. 10: 93 - 99 